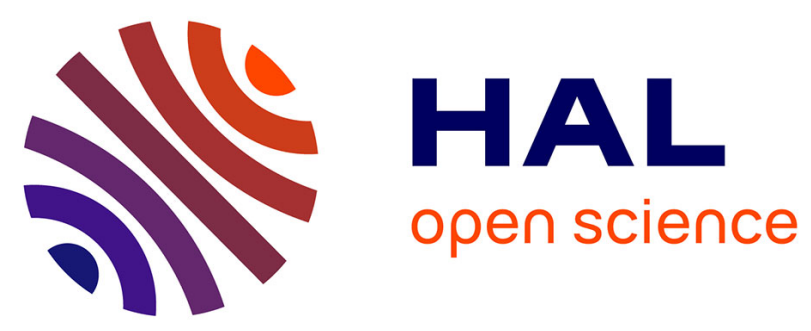

\title{
Towards Proactive Navigation: A Pedestrian-Vehicle Cooperation Based Behavioral Model
}

\author{
Maria Kabtoul, Anne Spalanzani, Philippe Martinet
}

\section{To cite this version:}

Maria Kabtoul, Anne Spalanzani, Philippe Martinet. Towards Proactive Navigation: A PedestrianVehicle Cooperation Based Behavioral Model. ICRA 2020 - IEEE International Conference on Robotics and Automation, May 2020, Paris, France. pp.6958-6964. hal-02509637

\section{HAL Id: hal-02509637 \\ https://hal.science/hal-02509637}

Submitted on 17 Mar 2020

HAL is a multi-disciplinary open access archive for the deposit and dissemination of scientific research documents, whether they are published or not. The documents may come from teaching and research institutions in France or abroad, or from public or private research centers.
L'archive ouverte pluridisciplinaire HAL, est destinée au dépôt et à la diffusion de documents scientifiques de niveau recherche, publiés ou non, émanant des établissements d'enseignement et de recherche français ou étrangers, des laboratoires publics ou privés. 


\title{
Towards Proactive Navigation: A Pedestrian-Vehicle Cooperation Based Behavioral Model
}

\author{
Maria Kabtoul $^{1}$, Anne Spalanzani ${ }^{1}$ and Philippe Martinet ${ }^{2}$
}

\begin{abstract}
Developing autonomous vehicles capable of navigating safely and socially around pedestrians is a major challenge in intelligent transportation. This challenge cannot be met without understanding pedestrians' behavioral response to an autonomous vehicle, and the task of building a clear and quantitative description of the pedestrian to vehicle interaction remains a key milestone in autonomous navigation research. As a step towards safe proactive navigation in a space shared with pedestrians, this work introduces a pedestrianvehicle interaction behavioral model. The model estimates the pedestrian's cooperation with the vehicle in an interaction scenario by a quantitative time-varying function. Using this cooperation estimation the pedestrian's trajectory is predicted by a cooperation-based trajectory planning model. Both parts of the model are tested and validated using real-life recorded scenarios of pedestrian-vehicle interaction. The model is capable of describing and predicting agents' behaviors when interacting with a vehicle in both lateral and frontal crossing scenarios.
\end{abstract}

\section{INTRODUCTION}

Developing navigation systems for autonomous vehicles in scenarios including interactions with vulnerable road users is becoming more and more interesting with the growing influence of the "Shared Space" concept in city planning across Europe [1]. Several studies have proven shared spaces to be a way of improving road users' safety and comfort [2].

Shared spaces introduce new dimensions to the navigation task making it an interdisciplinary challenge, which requires a study of the interaction between the vehicle and its conscious surrounding. Therefore, what was once regarded as a purely reactive task becomes a cooperative task between the vehicle and other agents in the space.

Cooperation has been shown to be a natural behavior in human societies on different scales [3], [4], and the task of navigating in a shared space can be viewed as a cooperative task, as it involves pooling and sharing of resources (space and time) between the pedestrians and the vehicle. Recently, [5] has presented some advancement in the domain of cooperative navigation. The work deals with the navigation task as a cooperative task between the robot and the human. The proposed method enables the robot to navigate in a number of indoor scenarios that would fail otherwise, albeit without presenting a quantitative measure of this cooperative behavior.

Furthermore, another challenge in cooperative navigation between a pedestrian and a vehicle is considering the psy-

\footnotetext{
${ }^{1}$ Univ. Grenoble Alpes, Inria, $38000 \quad$ Grenoanne.spalanzani@inria.fr

${ }^{2}$ Université Cote d'Azur, Inria, Sophia Antipolis, France philippe.martineteinria.fr
}

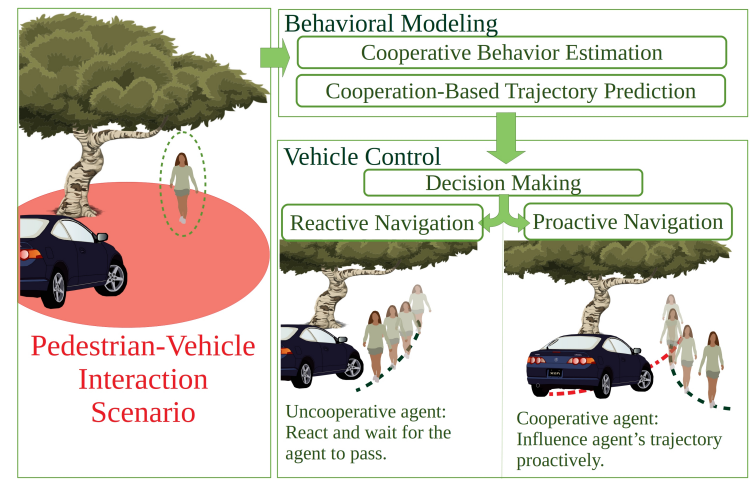

Fig. 1. Pedestrian-vehicle behavioral modeling as part of a global work scheme for proactive navigation

chology of the pedestrian. [6] constructed a framework for socially-aware navigation for any mobile robot starting from sociological concepts and proxemics theory. Applications requiring navigating around humans have exploited this framework to optimize the navigation task based on new social measures [7]. Including this aspect in the navigation scheme is crucial for autonomous vehicle's as well to ensure the integration of the latter in our daily life. However, there is an obvious lack of literature on studying human-vehicle interaction, cooperation and social rules, compared to that of human-robot interaction in general [8]. While the former falls under the same category, it is important to consider the particularity of the situation when working with an autonomous vehicle governed by its special properties and social conventions.

Some recent works have tackled the challenge of understanding and modeling the behavior of pedestrians around an autonomous vehicle. [9] formulates a model for pedestrians interaction based on social and psychological traits. This is done by assigning a dominance percentage to each pedestrian in an interaction scenario. The main limitation of this approach is that the model is based on pedestrian-pedestrian interaction and the assumption that this model will apply to pedestrian-vehicle interaction. However, the use of the social and psychological traits to build the model is inspiring and we construct our pedestrian-vehicle model in a similar manner. A different method to model pedestrian-vehicle interaction is based on extending the classical social force model (SFM). [10] extends the SFM by adding a repulsive force specific to the vehicle's influence. This resulted in a promising model, simulating several interaction scenarios in a shared space, however it was not validated on real-life data. 
In this work, a pedestrian-vehicle behavioral model is established, in order to be exploited later in a larger scheme for a proactive navigation application (Fig. 1). Proactive navigation is a major challenge on the way to integrating the autonomous vehicle in the shared space as an influential agent, taking an active role in the scene and not merely reacting to it.

This is achieved by first building a model capable of estimating the cooperative behavior of an agent in an interaction scenario with the vehicle (Behavioral Modeling in Fig. 1 . Afterwards, a decision is made on the ability and desirability of increasing the current cooperation of the agent. In other words, the vehicle either acts reactively or proactively.

The cooperation model is constructed based on social rules and cognitive studies [6], as well as the VCI-CITR ${ }^{1}$ dataset which includes top-view trajectory data of pedestrians in groups under vehicle influence in a shared space [11]. The evaluation of the pedestrians cooperation is then used to predict their trajectories. Later on, based on this cooperation model, the vehicle's controls can be computed to achieve a desired cooperative interaction.

The structure of this paper is as follows: Section II presents the main concepts and notations used in this work. Sections III and IV include the methodology of the pedestrian-vehicle behavioral modeling. Finally, section $\mathrm{V}$ discusses the main results of the work.

\section{BACKGRound, TERMS AND NotATIONS}

For modeling the interactions between the pedestrians, the concept of the personal zone is used. The personal zone (zone $P$ in Fig. 22 is a space around the pedestrian in which any human intrusion would cause discomfort. Different works may consider different shapes for the personal space around a pedestrian [6]. However, in this paper, a circular shape for all the zones is considered as the simplest case.

Inspired by the concept of the personal zone, we introduce the cooperation zone, which is a new zone specific to the pedestrian's interactions with a vehicle (zone $C$ in Fig. 2). As the pedestrian tends to clear the personal zone from human intrusion, (s)he tends to clear the cooperation zone of any vehicle intrusion.

Another concept used in building the model is the concept of the deformable virtual zone (DVZ). This concept was

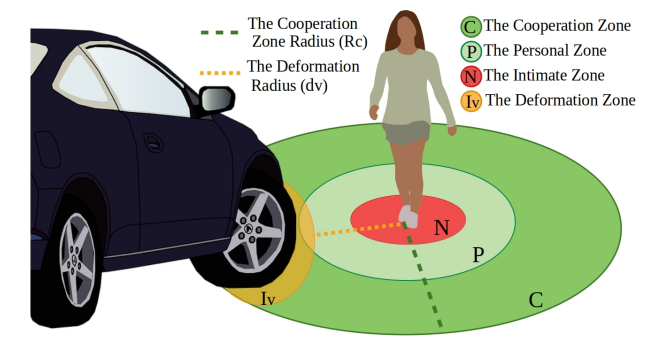

Fig. 2. The Deformation of the cooperation zone due to vehicle intrusion

${ }^{1}$ Vehicle-Crowd Interaction data-set - CITR lab: https://github. com/dongfang-steven-yang/vci-dataset-citr first introduced in [12] and it has been used since to model systems' maneuvers in both 2D and 3D spaces [13],[14],[15]. The idea is to surround the system under study with a virtual zone, and any body entering that zone will cause a deformation. The system can be driven in the direction minimizing this deformation or changing it in a desired way.

By applying this concept to the two previous zones, four parameters are defined: two scalars expressing the deformation of the zones and two angles expressing the mean orientation of the deformation of each zone with respect to the pedestrian. First, the deformation of the cooperation zone due to vehicle intrusion (zone $I_{V}$ in Fig. 2): a scalar $I_{V}$ :

$$
I_{V}(t)=\frac{1}{2 \pi} \int_{\alpha=0}^{2 \pi} \frac{D_{V}(\alpha, t)}{R_{C}} d \alpha
$$

where $R_{C}$ is the radius of the cooperation zone, and $D_{V}(\alpha, t)$ is the deformation at angle $\alpha$ and time $t$ :

$$
D_{V}(\alpha, t)= \begin{cases}R_{C}-d_{v}(\alpha, t) & \text { if } d_{v}(\alpha, t)<R_{C} \\ 0 & \text { Otherwise }\end{cases}
$$

and $d_{v}$ is the deformation radius computed as the distance between the pedestrian and the deformed zone, as shown in Fig. 2.

Secondly, the cooperation zone weighted deformation angle is defined as:

$$
\Theta_{V}(t)=\frac{\frac{1}{2 \pi} \int_{\alpha=0}^{2 \pi} D_{V}(\alpha, t) \alpha d \alpha}{I_{V}(t)}
$$

Finally, in a similar manner, $I_{P}^{a}(t)$ and $\Theta_{P}^{a}(t)$ are defined as the deformation of the personal zone and the deformation angle respectively, of an agent $a$ due to pedestrian intrusion.

\section{Pedestrian-Vehicle Cooperation Modeling}

To model the cooperative behavior, a Cooperation Factor $(C F)$ is assigned to each agent $a$ through a simulation $C F_{a}(t)$. A larger $C F$ indicates a more cooperative behavior from the vehicle's point of view. The goal is to find a model that expresses the cooperation of an agent $a$ as follows:

$$
C F_{a}(t)=f_{c}\left(P_{c f}^{a}(t)\right)
$$

where $f_{c}$ is a first order linear function:

$$
\begin{gathered}
f_{c}: \quad[0,1]^{4 \times 1} \rightarrow[0,1] \\
P_{c f}^{a} \longmapsto A P_{c f}^{a}+B
\end{gathered}
$$

$A \in \mathbb{R}^{1 \times 4}, B \in \mathbb{R}$, and the cooperation parameters of agent $a\left(P_{c f}^{a}\right)$ are the following:

$$
P_{c f}^{a}(t)=\left[\mathbb{P}_{[a, v e h]}(t), \mathcal{D}^{a}(t), I_{P}^{a}(t), \frac{V_{m}^{a}(t)}{V_{P \max }}\right]^{T}
$$

where: $\mathbb{P}_{[a, v e h]}(t)$ is the probability of collision of agent $a$ with the vehicle, $\mathcal{D}^{a}(t)$ is the density of the space surrounding the agent, $I_{P}^{a}(t)$ is the deformation of the personal zone around the agent, $V_{m}^{a}(t)$ is the mean velocity of the agent $\left(m . s^{-1}\right)$ and $V_{P \max }$ is the maximum allowed velocity of a pedestrian in the shared space. 
Our choice of parameters can be viewed as a time-varying extension and a new adaptation of the motion parameters proposed in [16] to the case of a human-vehicle interaction. Where the choice of $\mathbb{P}_{[a, v e h]}(t)$ is based on the straightforward correlation between a cooperative behavior of a pedestrian in interaction with a vehicle and the estimated threat of the situation. Moreover, $I_{P}^{a}(t)$ represents the tendency of humans to maintain their personal zone clear of interference [6] using the concept of the deformable virtual zone (DVZ) [12]. In the same time, $I_{P}^{a}(t)$ is a representation of the other parameters proposed in [16].

The model parameters: $\Phi=[A, B]$ are found by using a number of simulations including interactions between a group of pedestrians and a vehicle. A manual annotation is performed where a mean cooperation value is assigned to each agent in each simulation depending on the agent's behavior. First, knowing each agent's goal destination in the simulation, the agent's trajectory is observed and one of the four descriptions is assigned to this agent:

- Uncooperative Agent (UC): The agent took the optimal path to the goal point, without cooperating with the vehicle.

- Somewhat Cooperative Agent (SC): The agent modified the optimal path, assuming some cooperation on the vehicle's side.

- Highly Cooperative Agent (HC): The agent modified the optimal path, taking most of the burden in the pedestrian-vehicle cooperation task.

- Unidentified: cannot assign a clear cooperation description for the specific interaction case.

Afterwards, a mean cooperation value $(M C F)$ over the simulation time $(T)$ is assigned to each agent after discarding the unidentified cases:

$$
M C F(a)=\frac{1}{T} \int_{t=t_{0}}^{t_{0}+T} C F_{a}(t)= \begin{cases}0.2 & \text { if agent } a \text { is UC } \\ 0.5 & \text { if agent } a \text { is SC } \\ 0.8 & \text { if agent } a \text { is } \mathrm{HC}\end{cases}
$$

Fig. 3 shows an example of the cooperative behavior annotation. In Fig. 3 a the agent is uncooperative: the agent plans a straight path assuming the vehicle will move out of the way. Whereas, in Fig. 3 b the agent is somewhat cooperative: the planned path assumes some cooperation on the vehicle's side. Finally, in Fig. $3 \mathrm{c}$ the agent is highly cooperative: the planned path does not assume any cooperation on the vehicle's side.

The optimization problem is clearly under-constrained as a time-varying profile of the cooperation is extracted using a single value (the mean value of the function). The

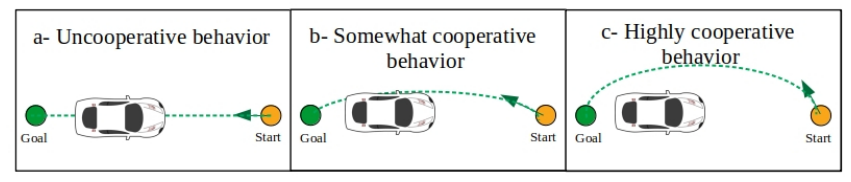

Fig. 3. Example of the cooperative behavior annotation. problem is further constrained by exploiting the fact that in each scenario the agents exhibiting similar cooperative behaviors are assigned similar nominal values. Meaning that the optimal $C F$ model should result in highly correlated profiles for agents with similar mean cooperation values in one simulation, and similarly, highly uncorrelated profiles for agents with contradicting cooperation behaviors (HC and UC).

Finally, the pedestrian-vehicle cooperation model is obtained by finding the optimal value for the model parameters $\Phi=[A, B]$ such that:

$$
\Phi=\arg \min _{\Phi} J_{1}(\Phi)+J_{2}(\Phi)
$$

where:

$$
J_{1}(\Phi)=\sum_{s=1}^{M} \sum_{a=1}^{A(s)}\left[M C F(a)-\frac{1}{T} \int_{t=t_{0}}^{t_{0}+T} f_{c}\left(P_{c f}^{a}(t), \Phi\right)\right]_{(9)}
$$

$M$ is the total number of simulations and $A(s)$ is the total number of agents in a simulation $s$, and $J_{2}$ is the term that maximizes the cross-correlation (CC) between the identically annotated profiles and minimizes it between the contradicting annotations in one simulation:

$$
J_{2}(\Phi)=\sum_{s=1}^{M} \sum_{k=1}^{A(s)} \sum_{l=1}^{A(s)} R_{C C}\left[C F_{k}, C F_{l}\right] \lambda(k, l)
$$

where $R_{C C}\left[C F_{k}, C F_{l}\right]$ is the cross-correlation factor between the cooperation factor profiles of agents $(l, k)$ :

$$
R_{C C}\left[C F_{k}, C F_{l}\right]=\frac{\int_{t=t_{0}}^{t_{0}+T} C F_{k}(t) * C F_{l}(t)}{\sqrt{\int_{t=t_{0}}^{t_{0}+T} C F_{k}(t)^{2} * \int_{t=t_{0}}^{t_{0}+T} C F_{l}(t)^{2}}}
$$

and:

$$
\lambda(k, l)= \begin{cases}0 & \text { if } k=l \\ -1 & \text { if } k \neq l \text { and } M C F(k)=M C F(l) \\ 1 & \text { if } k \neq l \text { and } M C F(k)=1-M C F(l)\end{cases}
$$

The output of the cooperation model is used next to predict the behavior of the agent in a particular situation.

\section{CoOperation-Based Pedestrians Trajectory PLANNING MODEL}

The main idea behind the model is that the trajectory of an agent can be predicted knowing two things: a- The state of the surrounding space which will impose some restrictions over the movement, and b- How cooperative this particular agent is.

The trajectory planning model is of the form:

$$
\vec{V}^{a}(t+1)=f_{T}\left(\vec{V}^{a}(t), P_{m}(t)\right)
$$

where $\vec{V}^{a}(t+1)$ and $\vec{V}^{a}(t) \in \mathbb{R}^{2}$ are the predicted and current velocity of agent $a$ respectively, and $f_{T}$ is a $C^{1}$ smooth function:

$$
\begin{gathered}
f_{T}: \quad \mathbb{R}^{N} \rightarrow\left[0, V_{P \max }\right] \times[0,2 \pi] \\
P_{m} \mapsto f_{T}\left(P_{m}\right)
\end{gathered}
$$


and the model motion parameters: $(N=7)$

$$
P_{m}(t)=\left[\begin{array}{c}
C F_{a}(t) \cdot I_{V}(t) \\
C F_{a}(t) \cdot \Theta_{V}(t) \\
{\left[1-C F_{a}(t)\right] \cdot \Theta_{\text {goal }}^{a}(t)} \\
{\left[1-C F_{a}(t)\right] \cdot D_{\text {goal }}^{a}(t)} \\
C \dot{F}_{a}(t) \\
I_{P}^{a}(t) \\
\Theta_{P}^{a}(t)
\end{array}\right]
$$

where: $\Theta_{\text {goal }}^{a}(t), D_{\text {goal }}^{a}(t)$ are the orientation and the distance of the agent's goal point at time $t$ respectively.

Multiplying the parameters resulting from the vehicle's influence $\left(I_{V}, \Theta_{V}\right)$ by the cooperation factor in the first two terms refers to the fact that the more cooperative the agents are, the more their trajectories are influenced by the vehicle. Similarly, the less cooperative the agents are, the more their trajectories are influenced by the goal destination. Hence, the destination parameters $\left(\Theta_{\text {goal }}^{a}, D_{\text {goal }}^{a}\right)$ are weighted by the inverse of the cooperation factor in the third and fourth terms. The remaining terms include the effect of the surrounding pedestrians and the change in the agent's cooperation.

\section{Simulations and Results}

Both the cooperation model and the cooperation-based trajectory planning model are focused on scenarios involving a group of pedestrians in interaction with a vehicle in a shared space. Meaning that the space does not constrain the movement of the pedestrians nor the vehicle, neither does it prioritize any party. The considered scenarios can include two types of interactions between a vehicle and a group of pedestrians: lateral and frontal crossing (Fig. 4). These two types present the most basic interactions that still occur frequently in a shared space.

The two models are estimated and validated using the VCI-CITR data-set [11]. The scenarios in the data-set include frontal and lateral crossing interactions between a group of pedestrians (7 to 10 in each simulation) and a vehicle. The set of scenarios used in this work consists of 22 simulations divided as follows:

- Frontal Crossing: 4 simulations.

- Lateral Crossing: 10 bidirectional, 4 unidirectional with a yield driving mode and 4 unidirectional with a normal driving mode.

Each one of the four previous types is divided into two sets. An estimation set with $75 \%$ of the total number of simulations in each type, and a validation set with the remaining $25 \%$.

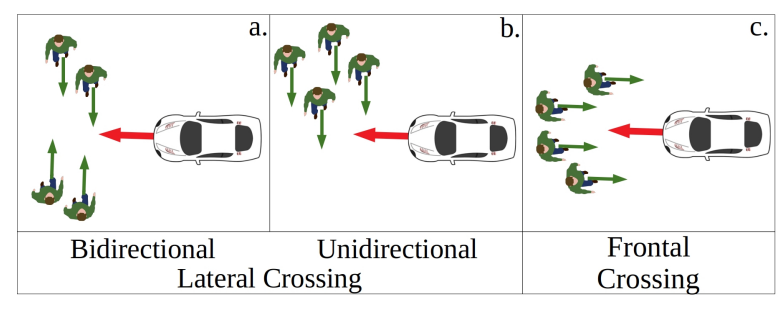

Fig. 4. Illustration of the pedestrians-vehicle interaction scenarios

\section{A. The pedestrian-vehicle cooperation model}

1) Model parameters estimation:

Using all the pedestrians-vehicle interaction scenarios selected from the data-set (both the estimation and validation sets), a mean cooperation value is assigned to each agent in each simulation depending on its behavior, as explained in section [II]

Afterwards, using only the data from the estimation set, a first order linear regression is used to fit the model in (4) to the annotated data. Meaning that the model parameters $\Phi$ are found using only the first optimization criterion: $J_{1}$ in (9). The result is then used as an initial condition for a derivative-free optimization based on the second criterion: adding $J_{2}$ in 10 to further restrict the model.

2) Model evaluation:

To evaluate the accuracy of the cooperation model, a crosscorrelation test is implemented. Where for each pair of agents in each simulation, the cross-correlation factor between their cooperation profiles is computed.

First, the ground-truth values of the $\mathrm{CC}$ factors $\left(R_{G T}\right)$ is obtained using the earlier annotations as shown in Table [ ]

\begin{tabular}{|c|l|}
\hline \multicolumn{2}{|c|}{ Cross-Correlation Factors Ground Truth } \\
\hline Agent Pair Annotation & $R_{G T}$ \\
\hline Similar behavior (SB): [UC, UC] or [HC, HC] or [SC, SC] & 1 \\
\hline Inverse Behavior (IB): [HC, UC] or [UC, HC] & 0 \\
\hline Unidentified Cases: [Nan, -] or [-, Nana] & Nan \\
\hline TABLE I
\end{tabular}

OBTAINING THE GROUND-TRUTH VALUES OF THE AGENTS CC FACTORS

After discarding the unidentified cases, the CC factors of all the agent pairs $\left(R_{C C}\right)$ are computed using (11). These continuous values are then discretized to be compared with the ground truth values:

$$
R_{C C}^{d}\left[C F_{k}, C F_{l}\right]= \begin{cases}1 & \text { if } R_{C C}\left[C F_{k}, C F_{l}\right] \geq 0.5 \\ 0 & \text { otherwise }\end{cases}
$$

and the behavior of the agent pair is predicted to be similar (SB) if $R_{C C}^{d}=1$, and inverse (IB) if $R_{C C}^{d}=0$.

Finally, the confusion matrix [17] is computed as illustrated in Table $\Pi$

\begin{tabular}{|c||c|c|}
\hline & Actual SB & Actual IB \\
\hline Predicted SB & True Similar $(t s)$ & False Inverse $(f i)$ \\
\hline Predicted IB & False Similar $(f s)$ & True Inverse $(t i)$ \\
\hline
\end{tabular}

TABLE II

OBTAINING THE CONFUSION MATRIX OF THE PREDICTED CC FACTORS

The accuracy of the model is evaluated using:

$$
\text { Accuracy }=\frac{t s+t i}{t s+f s+t i+f i} \times 100 \%
$$

The result of evaluating the confusion matrices and the accuracy of the model is shown in Table III for each type of the interaction scenarios in the data-set. 


\begin{tabular}{|c|c|c|c|c|c|c|}
\hline & \multicolumn{2}{|c|}{ Frontal Crossing } & \multicolumn{2}{|c|}{ Lateral Crossing } & \multicolumn{2}{|c|}{ All Scenarios } \\
\hline \multirow{2}{*}{$\begin{array}{r}\text { Confusion } \\
\text { Matrix }\end{array}$} & 44 & 0 & 224 & 4 & 268 & 4 \\
\hline & 4 & 5 & 60 & 50 & 64 & 55 \\
\hline Accuracy & \multicolumn{2}{|c|}{$92 \%$} & \multicolumn{2}{|c|}{$81 \%$} & \multicolumn{2}{|c|}{$83 \%$} \\
\hline
\end{tabular}

TABLE III

THE COOPERATION MODEL ACCURACY BASED ON THE CC TEST

The cooperation model is able to predict the similarities in agents' behaviors with a good accuracy in both lateral and frontal crossing scenarios. Noting that the available scenarios in the data-set provided a small sample of agent pairs with inverse behaviors in the frontal crossing simulations, which leads to an unreliable evaluation of the accuracy for the frontal crossing cases.

Fig. 5 shows an example of the cooperation factor profiles for a group of agents in a lateral crossing scenario. It can be observed in the figure that agents 1,6,7,8 show high cooperation factor values towards the end of the simulation $(C F>0.75)$ as these agents did not pass in front of the vehicle, but waited for it to pass instead. Agents 3,5 show low $\mathrm{CF}$ values $(C F<0.3)$ when passing in front of the vehicle while it is approaching. On the other hand, agent 2 shows higher $\mathrm{CF}$ values than agents 3,5 as it passed in front of the vehicle earlier in the simulation when it was further away. Finally, agent 4 would be expected to have a CF profile more similar to agent 6 , but its somewhat stable cooperation may be attributed to the fact that it experienced less overall

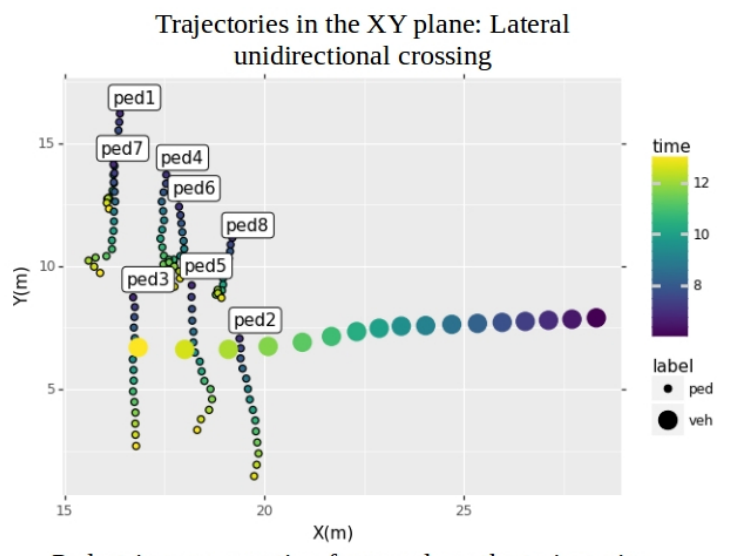

Pedestrians cooperation factors along the trajectories
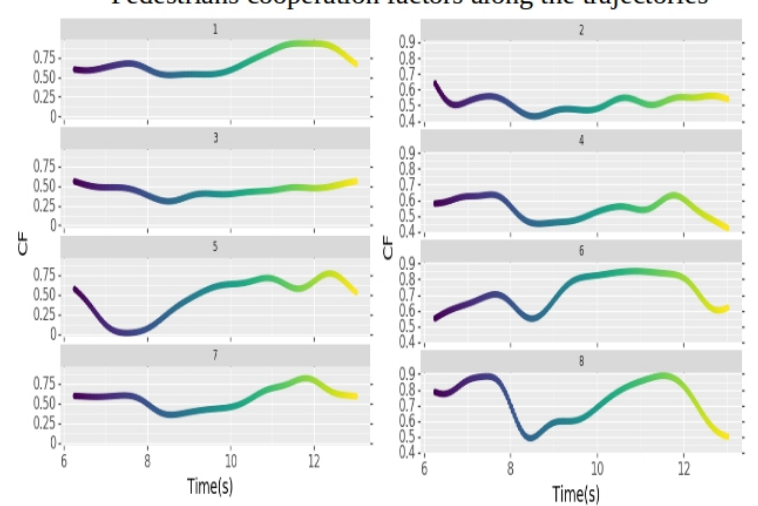

Fig. 5. The cooperation factor profiles of the agents in a lateral crossing scenario acceleration and was further away from the vehicle than agent 6 .

\section{B. The cooperation-based trajectory planning model}

\section{1) Model parameters estimation:}

Using the estimation set data, a linear regression is used to find the model parameters in 13 by minimizing the error between the model output and the trajectories in the estimation set.

The resulting model is of the form:

$$
\begin{gathered}
f_{T}: \quad \mathbb{R}^{7} \rightarrow\left[0, V_{P \max }\right] \times[0,2 \pi] \\
P_{m} \mapsto C P_{m}+E
\end{gathered}
$$

where: $C \in \mathbb{R}^{2 \times 7}, E \in \mathbb{R}^{2 \times 1}$

2) Model evaluation:

To evaluate the model, the Mean Square Error (MSE\%) between the trajectories predicted by the model and the real trajectories in the validation set is computed. Table IV] shows the $M S E \%$ in the linear velocity and the orientation for each type of interaction scenarios computed using:

$$
M S E \%=\frac{1}{M} \sum_{s=1}^{M} \frac{1}{A(s)} \sum_{a=1}^{A(s)} \frac{1}{T(s)} \sum_{t_{k}=t_{0}}^{T(s)+t_{0}} \frac{X_{\text {real }}^{a, s}\left(t_{k}\right)-X_{\text {model }}^{a, s}\left(t_{k}\right)}{X_{\text {real }}^{a, s}\left(t_{k}\right)} \times 100 \%
$$

where $M$ is the total number of simulations, $A(s)$ is the total number of agents in a simulation $s$ and $T(s)$ is the period of the simulation which serve as the prediction horizon. $X_{\text {real }}^{a, s}$, $X_{\text {model }}^{a, s}$ are the real and the model output values respectively for agent $a$ in simulation $s$, which is replaced by either the linear velocity or the orientation.

\begin{tabular}{|r||c|c|c|}
\hline & Frontal Crossing & Lateral Crossing & All Scenarios \\
\hline $\begin{array}{r}\text { Linear } \\
\text { Velocity }\end{array}$ & $18.42 \%$ & $22.61 \%$ & $21.91 \%$ \\
\hline Orientation & $2.66 \%$ & $1.5 \%$ & $1.69 \%$ \\
\hline
\end{tabular}

TABLE IV

$M S E \%$ BETWEEN THE MODEL OUTPUT AND THE REAL TRAJECTORIES IN THE VALIDATION SET

The model predicts the agents trajectories with a good accuracy for both the linear velocity and the orientation. A higher orientation MSE value is observed is the frontal crossing scenarios. Whereas, a higher linear velocity MSE is observed for the lateral crossing. This is caused by the agents experiencing more frequent orientation variations in a frontal crossing interaction, and more linear velocity variations in a lateral crossing interaction.

Fig. 6 shows an example of the trajectories resulting from the model compared to the real trajectories in a frontal crossing scenario. Despite having a low MSE in the orientation, the effect of the orientation estimation error is still quite noticeable on the resulting trajectories in the figure, as it is accumulative. This can be reduced by having a feedback on the estimation of the real orientation in a realtime simulation. 
The resulting trajectories in the XY plane in a frontal crossing scenario

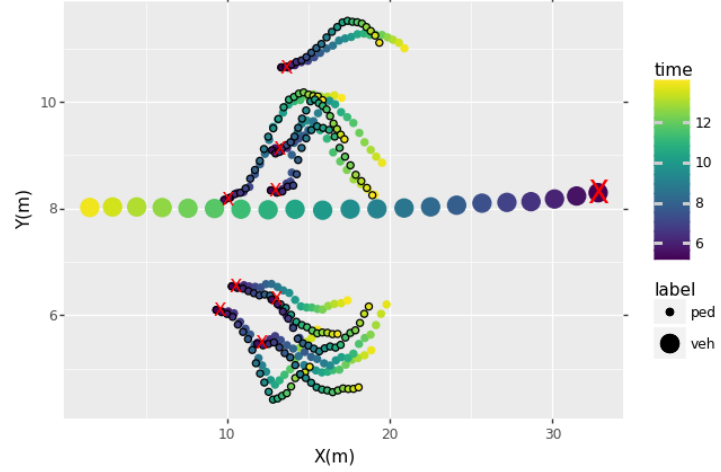

Fig. 6. Real and predicted trajectories in a frontal crossing scenario. Black contour: real, no contour: model output. X: starting point.

\section{Effect of changing the cooperation factor}

To visualize the effect of changing the cooperation factor of a group of agents in an interaction scenario, a reference frontal interaction simulation is generated using the cooperation model in (4). Afterwards, two other simulations are generated using two modified versions of the cooperation model: one with a $50 \%$ increment, and the other with a $50 \%$ decrements of the cooperation factor. Fig. 7 shows the three resulting simulations, where it can be observed that the simulation with an increased cooperation factor resulted in a more cooperative behavior on the agents side, moving out of the vehicle's way. Fig. 8 also shows the cooperation factors of the agents corresponding to the three simulations. Note that even-though an increment/decrement on the cooperation model is applied, the cooperation factor is still computed based on the evolution of the simulation.

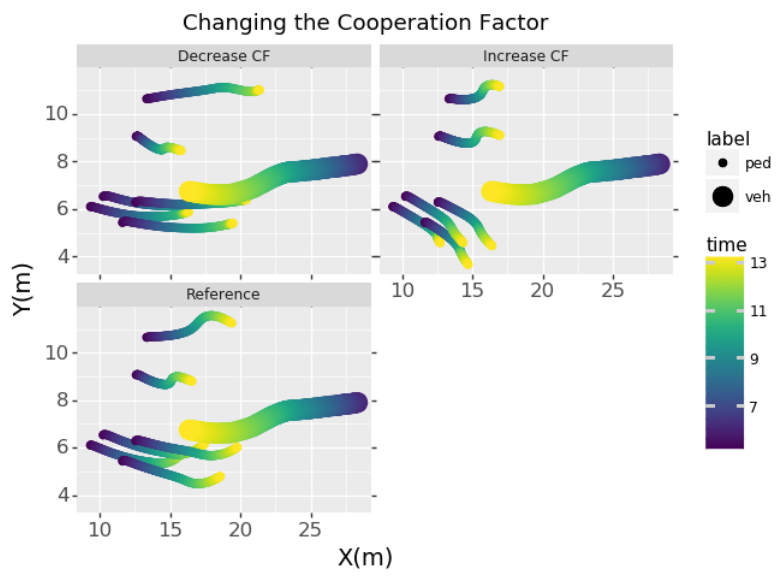

Fig. 7. Trajectories in the XY plane of the two simulations generated with increased/decreased cooperation factor values vs. the original reference simulation

\section{CONCLUSION}

In this work, we present a pedestrian-vehicle interaction model based on the concept of cooperative navigation, which can be exploited in autonomous navigation systems to ensure safe and socially-accepted behavior of the vehicle.
The cooperation estimation part of the model performs well in scenarios including a small group of pedestrians in interaction with a vehicle, and its output describes the change in the pedestrian's behavior using a time-varying cooperation factor ranging between zero (highly uncooperative) to one (highly cooperative). In future works, the ability of the cooperation model to identify groups of agents in dense spaces can be tested. The main limitation of this model is not considering other personal factors which cannot be measured directly from the state of the space, but can affect an individual's cooperation in real scenarios. For this reason, a variable parameter expressing the inner cooperation of a pedestrian can be added to the cooperation model in future works. This inner cooperation parameter can be estimated in real-time and its addition can help the model generalize to different types of interaction scenarios and reallife situations.

Furthermore, using the cooperation-based trajectory planning model, scenarios of lateral and frontal crossing of pedestrian-vehicle interaction can be simulated. The model is tested and verified using a set of real-life recorded scenarios. To our knowledge, this is the first attempt to validate a pedestrian-vehicle behavioral model on real scenarios, thanks to the release of the VCI-CITR data-set. Our future work will seek to generalize the presented method to more complex interactions that can occur in a shared space.
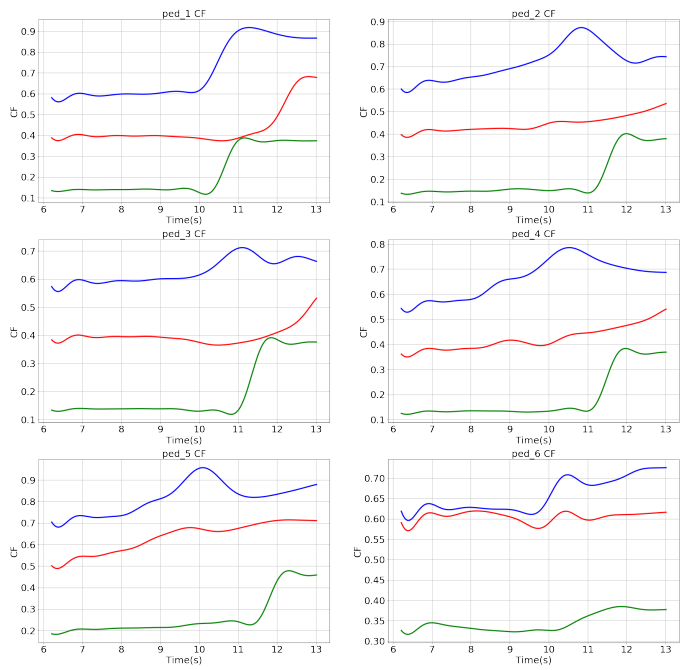

Fig. 8. The cooperation factors of the agents corresponding to the three simulations in Fig. 7 Red: reference simulation, blue: simulation generated with a $50 \%$ increment on $\mathrm{CF}$, green: simulation generated with a $50 \%$ decrement on CF.

\section{ACKNOWLEDGMENT}

This research has been conducted as part of the HIANIC (Human Inspired Autonomous Navigation In Crowds) project, funded by the French Ministry of Education and Research and the French National Research Agency (ANR-17-CE220010). 


\section{REFERENCES}

[1] B. Hamilton-Baillie, "Shared space: Reconciling people, places and traffic," Built Environment, vol. 34, pp. 161-181, May 2008.

[2] S. Moody and S. Melia, "Shared space - research, policy and problems," Proceedings of the ICE - Transport, vol. 167, pp. 384-392, Nov. 2014.

[3] R. Boyd and P. J. Richerson, "Cultural transmission and the evolution of cooperative behavior," Human Ecology, vol. 10, no. 3, pp. 325-351, Sep. 1982. [Online]. Available: https://doi.org/10.1007/BF01531189

[4] M. A. Nowak, "Five rules for the evolution of cooperation," Science, vol. 314, no. 5805, pp. 1560-1563, Dec. 2006. [Online]. Available: https://science.sciencemag.org/content/314/5805/1560

[5] H. Khambhaita and R. Alami, "A human-robot cooperative navigation planner," in Proceedings of the Companion of the 2017 ACM/IEEE International Conference on Human-Robot Interaction, ser. HRI '17. New York, NY, USA: ACM, 2017, pp. 161-162. [Online]. Available: http://doi.acm.org/10.1145/3029798.3038374

[6] J. Rios-Martinez, A. Spalanzani, and C. Laugier, "From proxemics theory to socially-aware navigation: A survey," International Journal of Social Robotics, vol. 7, pp. 137-153, Apr. 2014.

[7] T. Kruse, A. K. Pandey, R. Alami, and A. Kirsch, "Human-Aware Robot Navigation: A Survey," Robotics and Autonomous Systems, vol. 61, no. 12, pp. pp.1726-1743, Dec. 2013. [Online]. Available: https://hal.archives-ouvertes.fr/hal-01684295

[8] M. Goodrich and A. Schultz, "Human-robot interaction: A survey," Foundations and Trends in Human-Computer Interaction, vol. 1, pp. 203-275, Jan. 2007.

[9] T. Randhavane, A. Bera, E. Kubin, A. Wang, K. Gray, and D. Manocha, "Pedestrian dominance modeling for socially-aware robot navigation," CoRR, vol. abs/1810.06613, 2018. [Online]. Available: http://arxiv.org/abs/1810.06613

[10] D. Yang, U. Ozguner, and K. Redmill, "Social force based microscopic modeling of vehicle-crowd interaction," in 2018 IEEE Intelligent Vehicles Symposium (IV), Jun. 2018, pp. 1537-1542.

[11] D. Yang, L. Li, K. Redmill, and U. Ozguner, "Top-view trajectories: A pedestrian dataset of vehicle-crowd interaction from controlled experiments and crowded campus," 2019 IEEE
Intelligent Vehicles Symposium (IV), Jun. 2019. [Online]. Available: http://dx.doi.org/10.1109/IVS.2019.8814092

[12] R. Zapata, P. Lépinay, and P. Thompson, "Reactive behaviors of fast mobile robots," Journal of Robotic Systems, vol. 11, no. 1, pp. 13-20, 1994. [Online]. Available: https://onlinelibrary.wiley.com/doi/ abs/10.1002/rob.4620110104

[13] A. Cacitti and R. Zapata, "Reactive behaviours of mobile manipulators based on the dvz approach," in Proceedings 2001 ICRA. IEEE International Conference on Robotics and Automation (Cat. No.01CH37164), vol. 1, May 2001, pp. 680-685 vol.1.

[14] L. Amouri, C. Novales, G. Poisson, M. Njah, M. Jallouli, and N. Derbel, "DVZ-based Obstacle Avoidance Control of a Wheelchair Mobile Robot," in IEEE Intemational Conference on Mechatronics, Istanbul, Turkey, Apr. 2011, pp. pp 911-915. [Online]. Available: https://hal.archives-ouvertes.fr/hal-00630139

[15] G. Blanc, Y. Mezouar, and P. Martinet, "A path planning strategy for obstacle avoidance," in Proceedings of the Third International Conference on Informatics in Control, Automation and Robotics, vol. 2, INSTICC. SciTePress, 2006, pp. 438-444.

[16] S. J. Guy, S. Kim, M. C. Lin, and D. Manocha, "Simulating heterogeneous crowd behaviors using personality trait theory," in Proceedings of the 2011 ACM SIGGRAPH/Eurographics Symposium on Computer Animation, ser. SCA '11. New York, NY, USA: ACM, 2011, pp. 43-52. [Online]. Available: http://doi.acm.org/10. $1145 / 2019406.2019413$

[17] S. M. Hossin M, "A review on evaluation metrics for data classification evaluations," International Journal of Data Mining and Knowledge Management Process, vol. 5, no. 2, pp. 1-11, 2015. 